\title{
SOME PROPERTIES OF QUASI-ARMENDARIZ RINGS AND THEIR GENERALIZATIONS
}

\author{
ELTIYEB ALI*, AYOUB ELSHOKRY
}

Department of Mathematics, Faculty of Education, University of Khartoum, Omdurman, Sudan, P.O.Box 321

*Corresponding author: eltiyeb76@gmail.com

Received Sep 18, 2017

\begin{abstract}
Let $R$ be a ring and $(S, \leq)$ a strictly ordered monoid. The generalized power series ring $\left[\left[R^{S, \leq}\right]\right]$ with coefficients in $R$ and exponents in $S$ is a common generalization of polynomial rings, power series rings, Laurent polynomial rings, group rings, and MalcevNeumann Laurent series rings. We initiate the study of the $S$-quasi-Armendariz condition on $R$, a generalization of the standard quasi-Armendariz condition from polynomials to generalized power series. The class of quasi-Armendariz rings includes semiprime rings, Armendariz rings, right (left) p.q.-Baer rings and right (left) $P P$ rings. The $S$-quasi-Armendariz rings are closed under direct product. Also it is shown that, if $R$ is a left $A P P$-ring, then $R$ is $S$-quasi-Armendariz. The a necessary and sufficient condition is given for rings under which the ring $R$ is reflexive if and only if $\left[\left[R^{S, \leq]}\right]\right.$ is reflexive ring and $r_{\left[\left[R^{S, \leq}\right]\right]}\left(f\left[\left[R^{S, \leq}\right]\right]\right)$ is pure as a right ideal in $\left[\left[R^{S, \leq}\right]\right]$ for any element $f \in\left[\left[R^{S, \leq}\right]\right]$. We conclude some characterizations for generalized power series ring to be semiprime, quasi-Baer ring.

2010 Mathematics Subject Classification.16S99, 16W60, 16U80, 20M25.
\end{abstract}

Key words and phrases. generalized power series ring; $S$-quasi-Armendariz ring; semiprime ring; left $A P P$-ring; reflexive ring.

\section{Preliminaries}

All rings considered here are associative with identity. Any concept and notation not defined here can be found in Ribenboim ([17]-[20]), Elliott and Ribenboim [5]. We will write monoids multiplicatively unless otherwise indicated. If $R$ is a ring and $X$ is a nonempty subset of $R$, then the left (right) annihilator of $X$ in $R$ is denoted by $\ell_{R}(X)\left(r_{R}(X)\right)$.

Let $(S, \leq)$ be an ordered set. Recall that $(S, \leq)$ is artinian if every strictly decreasing sequence of elements of $S$ is finite, and that $(S, \leq)$ is narrow if every subset of pairwise order-incomparable elements of $S$ is finite. Thus, $(S, \leq)$ is artinian and narrow if and only if every nonempty subset of $S$ has at least one but only a finite number of minimal elements. Let 
$S$ be a commutative monoid. Unless stated otherwise, the operation of $S$ will be denoted additively, and the neutral element by 0 . The following definition is due to Elliott and Ribenboim [5].

Let $(S, \leq)$ is a strictly ordered monoid (that is, $(S, \leq)$ is an ordered monoid satisfying the condition that, if $s, s^{\prime}, t \in S$ and $s<s^{\prime}$, then $\left.s+t<s^{\prime}+t\right)$, and $R$ a ring. Let [[R $\left.R^{S, \leq]}\right]$ be the set of all maps $f: S \rightarrow R$ such that $\operatorname{supp}(f)=\{s \in S \mid f(s) \neq 0\}$ is artinian and narrow. With pointwise addition, $\left[\left[R^{S, \leq}\right]\right]$ is an abelian additive group. For every $s \in S$ and $f, g \in\left[\left[R^{S, \leq}\right]\right]$, let $X_{s}(f, g)=\{(u, v) \in S \times S \mid u+v=s, f(u) \neq 0, g(v) \neq 0\}$. It follows from Ribenboim $[20,4.1]$ that $X_{s}(f, g)$ is finite. This fact allows one to define the operation of convolution:

$$
(f g)(s)=\sum_{(u, v) \in X_{s}(f, g)} f(u) g(v)
$$

Clearly, $\operatorname{supp}(f g) \subseteq \operatorname{supp}(f)+\operatorname{supp}(g)$, thus by Ribenboim $[18,3.4] \operatorname{supp}(f g)$ is artinian and narrow, hence $f g \in\left[\left[R^{S, \leq}\right]\right]$. With this operation, and pointwise addition, $\left[\left[R^{S, \leq}\right]\right]$ becomes an associative ring, with identity element e, namely $e(0)=1, e(s)=0$ for every $0 \neq s \in S$. Which is called the ring of generalized power series with coefficients in $R$ and exponents in $S$. Many examples and results of rings of generalized power series are given in Ribenboim $([17]-[20])$, Elliott and Ribenboim [5] and Varadarajan ([12], [13]). For example, if $S=$

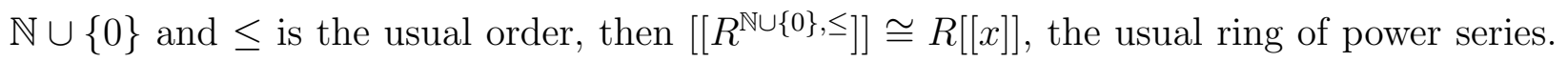
If $S$ is a commutative monoid and $\leq$ is the trivial order, then $\left[\left[R^{S, \leq}\right]\right] \cong R[S]$, the monoid ring of $S$ over $R$. Further examples are given in Ribenboim [18]. To any $r \in R$ and $s \in S$, we associate the maps $c_{r}, e_{s} \in\left[\left[R^{S, \leq}\right]\right]$ defined by

$$
c_{r}(x)=\left\{\begin{array}{ll}
r, & x=0, \\
0, & \text { otherwise }
\end{array} \quad e_{s}(x)= \begin{cases}1, & x=s \\
0, & \text { otherwise }\end{cases}\right.
$$

It is clear that $r \mapsto c_{r}$ is a ring embedding of $R$ into $\left[\left[R^{S, \leq}\right]\right], s \mapsto e_{s}$, is a monoid embedding of $S$ into the multiplicative monoid of the ring $\left[\left[R^{S, \leq}\right]\right]$, and $c_{r} e_{s}=e_{s} c_{r}$. Recall that a monoid $S$ is torsion-free if the following property holds: If $s, t \in S$, if $k$ is an integer, $k \geq 1$ and $k s=k t$, then $s=t$.

In this paper we give a new concept of $S$-quasi-Armendariz ring, which are a common generalization of quasi-Armendariz rings and $S$-Armendariz rings. We prove that, if $R$ is a left $A P P$-ring, then $R$ is $S$-quasi-Armendariz. Moreover, a ring $R$ is reflexive ring if and only if $\left[\left[R^{S, \leq}\right]\right]$ is reflexive ring and $\left.(1) r_{R}(a) R\right)$ is pure as a right ideal in $R$ for any element $a \in R ;(2) r_{\left[\left[R^{S, \leq}\right]\right]}\left(f\left[\left[R^{S, \leq}\right]\right]\right)$ is pure as a right ideal in $\left[\left[R^{S, \leq}\right]\right]$ for any element $f \in\left[\left[R^{S, \leq}\right]\right]$ in that case $R$ is $S$-quasi-Armendariz ring, where $(S, \leq)$ be a strictly ordered monoid. Also as a Corollary, a ring $R$ is a quasi-Baer ring if and only if $\left[\left[R^{S, \leq}\right]\right]$ is quasi-Baer ring and we give a 
lattice structure to the right (left) annihilators of a ring and characterize $S$-quasi-Armendariz rings as those rings $R$ for which an analogue of the Hirano map is a lattice isomorphism from the right (left) annihilators of $R$ to the right (left) annihilators of $\left[\left[R^{S, \leq}\right]\right]$.

\section{Generalization of quasi-Armendariz rings}

We start by the following definition:

Definition 2.1. Let $S$ be a torsion-free and cancellative monoid, $\leq$ a strict order on $S$. We say a ring $R, S$-quasi-Armendariz, if whenever $f, g \in\left[\left[R^{S, \leq}\right]\right]$ satisfy $f\left[\left[R^{S, \leq}\right]\right] g=0$, then $f(u) R g(v)=0$ for each $u, v \in S$.

The following result appeared in [24, Lemma 2.1].

Lemma 2.2. Let $S$ be a torsion-free and cancellative monoid, $\leq$ a strict order on $S$. Then $\left[\left[R^{S, \leq}\right]\right]$ is reduced if and only if $R$ is reduced.

Reduced rings are semicommutative. From Proposition 2.4 reduced rings are $S$-quasiArmendariz for any torsion free and cancellative monoid $S$. In [23, Corollary 2.3] it was claimed that all semicommutative rings are McCoy. However, Hirano's claim that, if $R$ is semicommutative then $R[x]$ is semicommutative, but this was later shown to be false in $[2$, Example 2]. Moreover, Nielsen [15] gave an example to show that a semicommutative ring $R$ need not be right McCoy, we also prove that the polynomial ring $R[x]$ over it actually is not semicommutative. By Liu [24], A ring $R$ is called $S$-Armendariz ring, if for each $f, g \in\left[\left[R^{S, \leq}\right]\right]$ such that $f g=0$ implies that $f(u) g(v)=0$ for each $u, v \in S$ and it was shown that generalized power series rings over semicommutative rings are semicommutative. Here we have the following.

Lemma 2.3. [24, Proposition 2.7] Let $(S, \leq)$ be a strictly ordered monoid and $R$ be an $S$-Armendariz ring. Then $R$ is semicommutative if and only if $\left[\left[R^{S, \leq}\right]\right]$ is semicommutative.

Proposition 2.4. Let $S$ be a torsion-free and cancellative monoid, $\leq$ a strict order on $S$ and $R$ a reduced ring. Then $R$ is an $S$-quasi-Armendariz.

Proof. Let $0 \neq f, g \in\left[\left[R^{S, \leq}\right]\right]$ be such that $f\left[\left[R^{S, \leq}\right]\right] g=0$. By Ribenboim [18], there exists a compatible strict total order $\leq^{\prime}$ on $S$, which is finer than $\leq$. We will use transfinite induction on the strictly totally ordered set $(S, \leq)$ to show that $f(u) R g(v)=0$ for any $u \in \operatorname{supp}(f)$ and $v \in \operatorname{supp}(g)$. Let $s$ and $t$ denote the minimum elements of $\operatorname{supp}(f)$ and $\operatorname{supp}(g)$ in the $\leq^{\prime}$ order, respectively. If $u \in \operatorname{supp}(f)$ and $v \in \operatorname{supp}(g)$ are such that $u+v=s+t$, then $s \leq^{\prime} u$ and $t \leq^{\prime} v$. If $s<^{\prime} u$ then $s+t<^{\prime} u+v=s+t$, a contradiction. Thus $u=s$. Similarly, $v=t$. Hence for any $r \in R, 0=\left(f c_{r} g\right)(s+t)=\sum_{(u, v) \in X_{s+t}\left(f, c_{r} g\right)} f(u) r g(v)=f(s) r g(t)$. 
Now suppose that $w \in S$ is such that for any $u \in \operatorname{supp}(f)$ and $v \in \operatorname{supp}(g)$ with $u+v<^{\prime}$ $w, f(u) R g(v)=0$. We will show that $f(u) R g(v)=0$ for any $u \in \operatorname{supp}(f)$ and $v \in \operatorname{supp}(g)$ with $u+v=w$. We write $X_{w}(f, g)=\{(u, v) \mid u+v=w, u \in \operatorname{supp}(f), v \in \operatorname{supp}(g)\}$ as $\left\{\left(u_{i}, v_{i}\right) \mid i=1,2, \ldots, n\right\}$ such that

$$
u_{1}<^{\prime} u_{2}<^{\prime} \cdots<^{\prime} u_{n} .
$$

Since $S$ is cancellative, $u_{1}=u_{2}$ and $u_{1}+v_{1}=u_{2}+v_{2}=w$ imply $v_{1}=v_{2}$. Since $\leq$ is a strict order, $u_{1}<^{\prime} u_{2}$ and $u_{1}+v_{1}=u_{2}+v_{2}=w$ imply $v_{2}<^{\prime} v_{1}$. Thus we have

$$
v_{n}<^{\prime} \cdots<^{\prime} v_{2}<^{\prime} v_{1} \text {. }
$$

Now, for any $r \in R$,

$$
0=\left(f c_{r} g\right)(w)=\sum_{(u, v) \in X_{w}\left(f, c_{r} g\right)} f(u) r g(v)=\sum_{i=1}^{n} f\left(u_{i}\right) r g\left(v_{i}\right) .
$$

For any $i \geq 2, u_{1}+v_{i}<^{\prime} u_{i}+v_{i}=w$, and thus, by induction hypothesis, we have $f\left(u_{1}\right) R g\left(v_{i}\right)=0$. Since $R$ is reduced, by Lemma 2.2 this implies $g\left(v_{i}\right) R f\left(u_{1}\right)=0$. Hence, multiplying (1) on the right by $f\left(u_{1}\right) g\left(v_{1}\right)$, we obtain

$$
\left(\sum_{i=1}^{n} f\left(u_{i}\right) r g\left(v_{i}\right)\right) f\left(u_{1}\right) g\left(v_{1}\right)=f\left(u_{1}\right) g\left(v_{1}\right) r f\left(u_{1}\right) g\left(v_{1}\right)=0 .
$$

Then $\left(f\left(u_{1}\right) r g\left(v_{1}\right)\right)^{2}=0$. Since $R$ is reduced, we have $f\left(u_{1}\right) r g\left(v_{1}\right)=0$. Now (1) becomes

$$
\sum_{i=2}^{n} f\left(u_{i}\right) r g\left(v_{i}\right)=0 .
$$

Multiplying $f\left(u_{2}\right) g\left(v_{2}\right)$ on (2) from the right-hand side, we obtain $f\left(u_{2}\right) r g\left(v_{2}\right)=0$ by the same way as the above. Continuing this process, we can prove $f\left(u_{i}\right) r g\left(v_{i}\right)=0$ for any $r \in R$, for $i=1,2, \ldots, n$. Thus $f(u) R g(v)=0$ for any $u \in \operatorname{supp}(f)$ and $v \in \operatorname{supp}(g)$ with $u+v=w$. Therefore, by transfinite induction, $f(u) R g(v)=0$ for any $u \in \operatorname{supp}(f)$ and $v \in \operatorname{supp}(g)$.

Corollary 2.5. [24, Lemma 3.1] Let $S$ be a torsion-free and cancellative monoid, $\leq$ a strict order on $S$, and $R$ a reduced ring. Then $R$ is $S$-Armendariz.

Proposition 2.6. Let $S$ be a torsion-free and cancellative monoid, $\leq$ a strict order on $S$. If $R$ is reduced semicommutative ring, then $R$ is $S$-Armendariz if and only if $R$ is $S$-quasiArmendariz.

Proof. Apply Lemma 2.3 and Proposition 2.4.

Proposition 2.7. Let $(S, \leq)$ be a strictly ordered monoid. Then every $S$-Armendariz rings are $S$-quasi-Armendariz. 
An ideal $I$ of $R$ is said to be right $s$-unital if, for each $a \in I$ there exists an element $e \in I$ such that $a e=a$. Note that if $I$ and $J$ are right $s$-unital ideals, then so is $I \cap J$ (if $a \in I \cap J$, then $a \in a I J \subseteq a(I \cap J))$.

The following result follows from Tominaga [11, Theorem 1].

Lemma 2.8. An ideal I of a ring $R$ is left (resp. right) s-unital if and only if for any finitely many elements $a_{1}, a_{2}, \ldots, a_{n} \in I$, there exists an element $e \in I$ such that $a_{i}=e a_{i}(r e s p$. $\left.a_{i}=a_{i} e\right)$ for each $i=1,2, \ldots, n$.

Clark defined quasi-Baer rings in [22]. A ring $R$ is called quasi-Baer if the left annihilator of every left ideal of $R$ is generated by an idempotent. Note that this definition is left-right symmetric. Some results of a quasi-Baer ring can be found in [16] and [22] and used them to characterize when a finite dimensional algebra with unity over an algebraically closed field is isomorphic to a twisted matrix units semigroup algebra. As a generalization of quasi-Baer rings, Birkenmeier, Kim and Park in [10] introduced the concept of principally quasi-Baer rings. A ring $R$ is called left principally quasi-Baer (or simply left p.q.-Baer) if the left annihilator of a principal left ideal of $R$ is generated by an idempotent. Similarly, right p.q.-Baer rings can be defined. A ring is called p.q.-Baer if it is both right and left p.q.Baer. Observe that biregular rings and quasi-Baer rings are p.q.-Baer. For more details and examples of left p.q.-Baer rings, see ([7]-[10]) and [27]. A ring $R$ is called a right (resp., left) $P$ P-ring if every principal right (resp., left) ideal is projective (equivalently, if the right (resp., left) annihilator of an element of $R$ is generated (as a right (resp., left) ideal) by an idempotent of $R$ ). A ring $R$ is called a $P P$-ring (also called a Rickart ring [3, p. 18]) if it is both right and left $P P$. We say a ring $R$ is a left $A P P$-ring if the left annihilator $l_{R}(R a)$ is right $s$-unital as an ideal of $R$ for any element $a \in R$. This concept is a common generalization of left p.q.-Baer rings and right $P P$-rings

Proposition 2.9. Let $(S, \leq)$ a strictly totally ordered monoid. If $R$ is left APP-ring, then $R$ is $S$-quasi-Armendariz.

Proof. Let $0 \neq f, g \in\left[\left[R^{S, \leq}\right]\right]$ be such that $f\left[\left[R^{S, \leq}\right]\right] g=0$. We use the transfinite induction to show that $f(u) R g(v)=0$ for all $u, v \in S$. Assume that $\pi(f)=u_{0}, \pi(g)=v_{0}$. Let $(u, v) \in X_{u_{0}+v_{0}}(f, g)$. So $u_{0} \leq u$ and $v_{0} \leq v$. If $u_{0}<u$, then $u_{0}+v_{0}<u+v_{0} \leq u+v=u_{0}+v_{0}$, a contradiction. Thus $u=u_{0}$. Similarly, $v=v_{0}$. So $X_{u_{0}+v_{0}}(f, g)=\left\{\left(u_{0}, v_{0}\right)\right\}$. Hence for any $r \in R$, from $f\left[\left[R^{S, \leq}\right]\right] g=0$ we have,

$$
0=\left(f c_{r} g\right)\left(u_{0}+v_{0}\right)=\sum_{(u, v) \in X_{u_{0}+v_{0}}\left(f, c_{r} g\right)} f(u) r g(v)=f\left(u_{0}\right) r g\left(v_{0}\right) \text {. }
$$


So $f\left(u_{0}\right) R g\left(v_{0}\right)=0$. Now, let $\lambda \in S$ with $u_{0}+v_{0} \leq \lambda$ and assume that for any $u \in \operatorname{supp}(f)$ and any $v \in \operatorname{supp}(g)$, if $u+v<\lambda$, then $f(u) R g(v)=0$. We claim that $f(u) R g(v)=0$, for each $u \in \operatorname{supp}(f)$ and each $v \in \operatorname{supp}(g)$ with $u+v=\lambda$. For convenience, we write $X_{\lambda}(f, g)=\{(u, v) \mid u+v=\lambda, u \in \operatorname{supp}(f), v \in \operatorname{supp}(g)\}$ as $\left\{\left(u_{i}, v_{i}\right) \mid i=1,2, \ldots, n\right\}$ such that

$$
u_{1}<u_{2}<\cdots<u_{n}
$$

where $n$ is a positive integer (Note that if $u_{1}=u_{2}$, then from $u_{1}+v_{1}=u_{2}+v_{2}$ we have $v_{1}=v_{2}$, and then $\left.\left(u_{1}, v_{1}\right)=\left(u_{2}, v_{2}\right)\right)$. Since $f\left[\left[R^{S, \leq}\right]\right] g=0$, for any $r \in R$ we have:

$$
0=\left(f c_{r} g\right)(\lambda)=\sum_{(u, v) \in X_{\lambda}\left(f, c_{r} g\right)} f(u) r g(v)=\sum_{i=1}^{n} f\left(u_{i}\right) r g\left(v_{i}\right) .
$$

Let $e_{u_{1}} \in r_{R}\left(f\left(u_{1}\right) R\right)$. So $f\left(u_{1}\right) R e_{u_{1}}=0$ and which implies $f\left(u_{1}\right) R e_{u_{1}} g\left(v_{1}\right)=0$. Let $r^{\prime} \in R$ be an arbitrary element. Then we have $f\left(u_{1}\right) r^{\prime} e_{u_{1}} g\left(v_{1}\right)=0$. Take $r=r^{\prime} e_{u_{1}}$ in Eq. (3). Thus,

$$
\sum_{i=2}^{n} f\left(u_{i}\right) r^{\prime} e_{u_{1}} g\left(v_{i}\right)=0
$$

Note that $u_{1}+v_{i}<u_{i}+v_{i}=\lambda$ for any $i \geq 2$. So by compatibility and induction hypothesis, $f\left(u_{1}\right) R g\left(v_{i}\right)=0$ for each $i \geq 2$. Since $R$ is right $A P P, r_{R}\left(f\left(u_{1}\right) R\right)$ is left $s$-unital. So without lose of generality and using Lemma 2.8, we can assume that $g\left(v_{i}\right)=e_{u_{1}} g\left(v_{i}\right)$, for each $i \geq 2$. Therefore

$$
\sum_{i=2}^{n} f\left(u_{i}\right) r^{\prime} g\left(v_{i}\right)=0
$$

Let $e_{u_{2}} \in r_{R}\left(f\left(u_{2}\right) R\right)$. So $f\left(u_{2}\right) R e_{u_{2}}=0$ and then $f\left(u_{2}\right) R e_{u_{2}} g\left(v_{2}\right)=0$. This implies $f\left(u_{2}\right) R e_{u_{2}} g\left(v_{2}\right)=0$.

Let $p \in R$ be an arbitrary element. So $f\left(u_{2}\right) p e_{u_{2}} g\left(v_{2}\right)=0$. Also note that $u_{2}+v_{i}<u_{i}+v_{i}=$ $\lambda$ for any $i \geq 3$. So by induction hypothesis, $f\left(u_{2}\right) R g\left(v_{i}\right)=0$. Therefore $g\left(v_{i}\right) \in r_{R}\left(f\left(u_{2}\right) R\right)$, for each $i \geq 3$. Since $r_{R}\left(f\left(u_{2}\right) R\right)$ is left $s$-unital, without lose of generality and using Lemma 2.8 , again we can assume that $g\left(v_{i}\right)=e_{u_{2}} g\left(v_{i}\right)$, for each $i \geq 3$. Take $r^{\prime}=p e_{u_{2}}$ in Eq. (4), so we have:

$$
\sum_{i=2}^{n} f\left(u_{i}\right) p e_{u_{2}} g\left(v_{i}\right)=0 .
$$

Continuing in this manner, we have $f\left(u_{n}\right) q g\left(v_{n}\right)=0$, where $q$ is an arbitrary element of $R$. Thus $f\left(u_{n}\right) R g\left(v_{n}\right)=0$. Hence $f\left(u_{n-1}\right) R g\left(v_{n-1}\right)=0, \ldots, f\left(u_{2}\right) R g\left(v_{2}\right)=0, f\left(u_{1}\right) R g\left(v_{1}\right)=$ 0 . Therefore, by transfinite induction, $f(u) R g(v)=0$ for any $u, v \in S$, and the proof is complete.

Corollary 2.10. Let $(S, \leq)$ a strictly totally ordered monoid. If I is a finitely generated left ideal of $R$ then for all $a \in l_{R}(I), a \in a l_{R}(I)$. So $R$ is $S$-quasi-Armendariz. 
Proof. By Proposition 2.9 and [26, Proposition 2.6].

Proposition 2.11. Let $S$ be a torsion-free and cancellative monoid, $\leq$ a strict order on $S$ and $R$ an $S$-quasi-Armendariz ring. If $f_{1}, \ldots, f_{n} \in\left[\left[R^{S, \leq}\right]\right] \triangleq \Lambda$ are such that $f_{1} \Lambda f_{2} \Lambda \cdots \Lambda f_{n}=$ 0 , then $f_{1}\left(u_{1}\right) R f_{2}\left(u_{2}\right) R \cdots R f_{n}\left(u_{n}\right)=0$ for all $u_{1}, u_{2}, \ldots, u_{n} \in S$.

Proof. Assume that $f_{1} \Lambda f_{2} \Lambda \cdots \Lambda f_{n}=0$. Then for any $g_{2}, g_{3}, \ldots, g_{n-1} \in \Lambda$,

$$
f_{1} \Lambda\left(f_{2} g_{2} \cdots g_{n-1} f_{n}\right)=0 .
$$

Since $R$ is $S$-quasi-Armendariz, we have

$$
f_{1}\left(u_{1}\right) R\left(\left(f_{2} g_{2} \cdots g_{n-1} f_{n}\right)(v)\right)=0
$$

for any $u_{1}, v \in S$. Thus

$$
\left(C_{f_{1}\left(u_{1}\right) r_{1}}\left(f_{2} g_{2} \cdots g_{n-1} f_{n}\right)\right)(v)=0
$$

for any $r_{1} \in R$ and any $v \in S$. So $C_{f_{1}\left(u_{1}\right) r_{1}} f_{2} g_{2} \cdots g_{n-1} f_{n}=0$, therefore $C_{f_{1}\left(u_{1}\right) r_{1}} f_{2} \Lambda \cdots$ $\Lambda f_{n}=0$, for any $r_{1} \in R$. Thus

$$
\left(C_{f_{1}\left(u_{1}\right) r_{1}} f_{2}\right) \Lambda\left(f_{3} g_{3} \cdots g_{n-1} f_{n}\right)=0 .
$$

By the hypothesis, we have

$$
\left(C_{f_{1}\left(u_{1}\right) r_{1}} f_{2}\right)\left(u_{2}\right) R\left(f_{3} g_{3} \cdots g_{n-1} f_{n}\right)(z)=0
$$

for any $u_{2}, z \in S$. Yields

$$
f_{1}\left(u_{1}\right) r_{1} f_{2}\left(u_{2}\right) R\left(f_{3} g_{3} \cdots g_{n-1} f_{n}\right)(z)=0 .
$$

So $f_{1}\left(u_{1}\right) r_{1} f_{2}\left(u_{2}\right) r_{2}\left(f_{3} g_{3} \cdots g_{n-1} f_{n}\right)(z)=0$, for any $r_{1}, r_{2}, \in R$. Thus

$$
\left(C_{f_{1}\left(u_{1}\right) r_{1} f_{2}\left(u_{2}\right) r_{2}} f_{3} g_{3} \cdots g_{n-1} f_{n}\right)(z)=0,
$$

for any $r_{1}, r_{2}, \in R$ and any $z \in S$. So $C_{f_{1}\left(u_{1}\right) r_{1} f_{2}\left(u_{2}\right) r_{2}} f_{3} g_{3} \cdots g_{n-1} f_{n}=0$ for any $g_{3}, \ldots, g_{n-1} \in$ $\Lambda$. Thus,

$$
C_{f_{1}\left(u_{1}\right) r_{1} f_{2}\left(u_{2}\right) r_{2}} f_{3} \Lambda \cdots \Lambda f_{n}=0 .
$$

Since $R$ is $S$-quasi-Armendariz. Repeating this process, we can get

$$
C_{f_{1}\left(u_{1}\right) r_{1} f_{2}\left(u_{2}\right) r_{2} \cdots r_{n-1} f_{n}\left(u_{n}\right)}=0 .
$$

So $f_{1}\left(u_{1}\right) r_{1} f_{2}\left(u_{2}\right) r_{2} \cdots r_{n-1} f_{n}\left(u_{n}\right)=0$ for any $u_{1}, u_{2}, \ldots, u_{n} \in S$ and any $r_{1}, r_{2}, \ldots$, $r_{n-1} \in R$. Therefore $f_{1}\left(u_{1}\right) R f_{2}\left(u_{2}\right) R \cdots R f_{n}\left(u_{n}\right)=0$ for any $u_{1}, u_{2}, \ldots, u_{n} \in S$.

The following is a generalization of Proposition 2.4. 
Corollary 2.12. Let $S$ be a torsion-free and cancellative monoid, $\leq$ a strict order on $S$ and $R$ a reduced ring. If $f_{1}, \ldots, f_{n} \in\left[\left[R^{S, \leq}\right]\right] \triangleq \Lambda$ are such that $f_{1} \Lambda f_{2} \Lambda \cdots \Lambda f_{n}=0$, then $f_{1}\left(u_{1}\right) R f_{2}\left(u_{2}\right) R \ldots R f_{n}\left(u_{n}\right)=0$ for all $u_{1}, u_{2}, \ldots, u_{n} \in S$.

Proposition 2.13. Let $S$ be a torsion-free and cancellative monoid, $\leq$ a strict order on $S$, and $R$ a reduced ring. Then $f R g=0$ if and only if $f\left[\left[R^{S, \leq}\right]\right] g=0$.

Proof. $(\Rightarrow)$ Assume that $0 \neq f, g \in\left[\left[R^{S, \leq}\right]\right]$ are such that $f R g=0$. By Corollary $2.5, R$ is $S$-Armendariz, so for any $h \in\left[\left[R^{S, \leq}\right]\right]$ and any $s \in S$,

$$
(f h g)(s)=\sum_{(u, w, v) \in X_{s}(f, h, g)} f(u) h(w) g(v)=0 .
$$

Thus $f h g=0$. This show that $f\left[\left[R^{S, \leq}\right]\right] g=0$. The "only if part" is clear.

According to [6], a right ideal $I$ is reflexive if $x R y \in I$ implies $y R x \in I$ for $x, y \in R$. Hence we shall call a ring $R$ a reflexive ring if 0 is a reflexive ideal (i.e., $a R b=0$ implies $b R a=0$ for $a, b \in R$ ). Moreover, a right ideal $I$ is called completely reflexive if $x y \in I$ implies $y x \in I$. A ring $R$ is completely reflexive if (0) has the corresponding property. It is clear that every completely reflexive ring is reflexive.

Proposition 2.14. Let $(S, \leq)$ be a strictly totally ordered monoid and $R$ be an $S$-quasiArmendariz ring. Then $R$ is reflexive ring if and only if $\left[\left[R^{S, \leq}\right]\right]$ is reflexive ring.

Proof. $(\Rightarrow)$ Let $R$ be reflexive ring. Suppose that $f, g \in\left[\left[R^{S, \leq}\right]\right]$ are such that $f\left[\left[R^{S, \leq}\right]\right] g=0$. Since $R$ is $S$-quasi-Armendariz, we have $f(u) R g(v)=0$ for any $u \in \operatorname{supp}(f)$ and $v \in \operatorname{supp}(g)$. But $R$ is reflexive, so $g(v) R f(u)=0$ for all $u, v \in S$. Now for any $h \in\left[\left[R^{S, \leq}\right]\right]$ and any $s \in S$,

$$
(g h f)(s)=\sum_{(v, w, u) \in X_{s}(g, h, f)} g(v) h(w) f(u)=0 .
$$

Thus $g h f=0$. This show that $g\left[\left[R^{S, \leq}\right]\right] f=0$. This means that $\left[\left[R^{S, \leq}\right]\right]$ is reflexive. $(\Leftarrow)$ Let $a, b \in R$ be such that $a R b=0$. Then $C_{a}\left[\left[R^{S, \leq}\right]\right] C_{b}=0$. Hence $C_{b}\left[\left[R^{S, \leq}\right]\right] C_{a}=0$ by reflexive. So $b R a=0$. Therefore $R$ is reflexive.

Corollary 2.15. Let $(S, \leq)$ be a strictly totally ordered monoid and $R$ a reduced ring. Then $R$ is reflexive ring if and only if $\left[\left[R^{S,} \leq\right]\right]$ is reflexive.

Due to Hirano [23]. A ring $R$ is called quasi-Armendariz provided that $a_{i} R b_{j}=0$ for all $i, j$ whenever $f(x)=a_{0}+a_{1} x+\cdots+a_{n} x^{n}, g(x)=b_{0}+b_{1} x+\cdots+b_{m} x^{m} \in R[x]$ satisfy $f(x) R[x] g(x)=0$.

Corollary 2.16. [14, Proposition 3.2] Let $R$ be a quasi-Armendariz ring, then the following statements are equivalent: 
(1) $R$ is reflexive.

(2) $R[x]$ is reflexive.

(3) $R\left[x ; x^{-1}\right]$ is reflexive.

A ring $R$ is called semiprime if for any $a \in R, a R a=0$, implies $a=0$. Let $R$ be a ring and $(S, \leq)$ a strictly totally ordered monoid. A ring $R$ is called $S$-semiprime if $f\left[\left[R^{S, \leq}\right]\right] f=0$, then $f=0$ for each $f \in\left[\left[R^{S, \leq}\right]\right]$.

The following result appeared in [25, Lemma 2.7]

Lemma 2.17. Let $R$ be a ring and $(S, \leq)$ a strictly totally ordered monoid. Then $R$ is a semiprime ring if and only if $\left[\left[R^{S, \leq}\right]\right]$ is a semiprime ring.

Proposition 2.18. Let $(S, \leq)$ be a strictly totally ordered monoid. If $R$ is a semiprime, then $R$ is $S$-quasi-Armendariz.

Proof. It follows from Proposition 2.9.

Corollary 2.19. If $S$ be a commutative, torsion-free, and cancellative monoid, then every semiprime ring $R$ is $S$-quasi-Armendariz.

Corollary 2.20. [23, Corollary 3.8] A semiprime ring is a quasi-Armendariz ring.

Corollary 2.21. Let $R$ be a ring and $(S, \leq)$ a strictly totally ordered monoid. If $R$ is semiprime, then $\left[\left[R^{S, \leq}\right]\right]$ is $S$-quasi-Armendariz ring.

Corollary 2.22. Let $R$ be a ring and $(S, \leq)$ a strictly totally ordered monoid. Assume that $R$ is semiprime. Then $R$ is reflexive ring if and only if $\left[\left[R^{S, \leq}\right]\right]$ is reflexive.

Theorem 2.23. Let $S$ be a torsion-free and cancellative monoid, $\leq$ a strict order on $S$. Then the following conditions are equivalent:

(1) $R$ is semiprime;

(2) $R$ is reduced $S$-quasi-Armendariz.

Proof. (1) $\Rightarrow(2)$ Is trivial.

$(2) \Rightarrow(1)$ Let $R$ be a reduced $S$-quasi-Armendariz. In particular for any $0 \neq f \in\left[\left[R^{S, \leq}\right]\right]$ be such that $f\left[\left[R^{S, \leq}\right]\right] f=0$, then $f(u) R f(u)=0$. Thus, $(R f(u))^{2}=0$ since $R$ is reduced. Therefore $f(u)=0$.

Let $I$ be an index set and $R_{i}$ be a ring for each $i \in I$. Let $(S, \leq)$ be a strictly ordered monoid, if there is an injective homomorphism $f: R \rightarrow \prod_{i \in I} R_{i}$ such that, for each $j \in I, \pi_{j} f: R \rightarrow R_{j}$ is a surjective homomorphism, where $\pi_{j}: \prod_{i \in I} R_{i} \rightarrow R_{j}$ is the $j$ th projection. We have the following. 
Proposition 2.24. Let $R_{i}$ be a ring, $(S, \leq)$ a strictly totally ordered monoid, for each $i$ in a finite index set I. If $R_{i}$ is $S$-quasi-Armendariz for each $i$, then $R=\prod_{i \in I} R_{i}$ is $S$-quasiArmendariz.

Proof. Let $R=\prod_{i \in I} R_{i}$ be the direct product of rings $\left(R_{i}\right)_{i \in I}$ and $R_{i}$ is $S$-quasi-Armendariz for each $i \in I$. Denote the projection $R \rightarrow R_{i}$ as $\Pi_{i}$. Suppose that $f, g \in\left[\left[R^{S, \leq}\right]\right]$ are such that $f\left[\left[R^{S, \leq}\right]\right] g=0$. Set $f_{i}=\prod_{i} f, g_{i}=\prod_{i} g$ and $h_{i}=\prod_{i} h$. Then $f_{i}, g_{i} \in\left[\left[R_{i}^{S, \leq}\right]\right]$. For any $u, v \in S$, assume $f(u)=\left(a_{i}^{u}\right)_{i \in I}, g(v)=\left(b_{i}^{v}\right)_{i \in I}$. Now, for any $h \in\left[\left[R^{S, \leq}\right]\right]$, any $r \in R$ and any $s \in S$,

$$
\begin{aligned}
\left(f c_{r} g\right)(s) & =\sum_{(u, v) \in X_{s}\left(f, c_{r} g\right)} f(u) r g(v) \\
& \left.=\sum_{(u, v) \in X_{s}\left(f, c_{r} g\right)}\left(a_{i}^{u}\right)_{i \in I}\left(r_{i}\right)\right)_{i \in I}\left(b_{i}^{v}\right)_{i \in I} \\
& =\sum_{(u, v) \in X_{s}\left(f, c_{r} g\right)}\left(\left(a_{i}^{u}\right) r_{i}\left(b_{i}^{v}\right)\right)_{i \in I} \\
& =\sum_{(u, v) \in X_{s}\left(f, c_{r} g\right)}\left(f_{i}(u) r_{i} g_{i}(v)\right)_{i \in I} \\
& =\left(\sum_{(u, v) \in X_{s}\left(f_{i}, c_{r_{i}} g_{i}\right)} f_{i}(u) r_{i} g_{i}(v)\right)_{i \in I} \\
& =\left(\left(f_{i} h_{i} g_{i}\right)(s)\right)_{i \in I} .
\end{aligned}
$$

Since $\left(f c_{r} g\right)(s)=0$ we have

$$
\left(f_{i} c_{r_{i}} g_{i}\right)(s)=0 .
$$

Thus, $f_{i} h_{i} g_{i}=0$. Now it follows $f_{i}(u) r_{i} g_{i}(v)=0$ for any $r \in R$, any $u, v \in S$ and any $i \in I$, since $R_{i}$ is $S$-quasi-Armendariz. Hence, for any $u, v \in S$,

$$
f(u) r g(v)=\left(f_{i}(u)\left(r_{i}\right) g_{i}(v)\right)_{i \in I}=0
$$

since $I$ is finite. Thus, $f(u) R g(v)=0$. This means that $R$ is $S$-quasi-Armendariz.

\section{Characterizations generalized power series quasi-Armendariz rings via annihilators}

In this section we give a lattice structure to the right (left) annihilators of a ring and characterize $S$-quasi-Armendariz rings as those rings $R$ for which an analogue of the Hirano [23] map is a lattice isomorphism from the right (left) annihilators of $R$ to the right (left) annihilators of $\left[\left[R^{S, \leq}\right]\right]$.

Let $\gamma=C(f)$ be the content of $f$, i.e., $C(f)=\{f(u) \mid u \in \operatorname{supp}(f)\} \subseteq R$. Since, $R \simeq c_{R}$ we can identify, the content of $f$ with

$$
c_{C(f)}=\left\{c_{f\left(u_{i}\right)} \mid u_{i} \in \operatorname{supp}(f)\right\} \subseteq\left[\left[R^{S, \leq}\right]\right] .
$$


Lemma 3.1. [21, Lemma 2.1] Let $R$ be a ring, $S$ a strictly ordered monoid, $\left[\left[R^{S, \leq}\right]\right]$ the generalized power series ring and $U \subseteq R$. Then

$$
\left[\left[R^{S, \leq}\right]\right] \ell_{R}(U)=\ell_{\left[\left[R^{S, \leq]}\right]\right.}(U),\left(r_{R}(U)\left[\left[R^{S, \leq}\right]\right]=r_{\left[\left[R^{S, \leq}\right]\right]}(U)\right) .
$$

By Lemma 3.1 we have two maps $\phi: r A n n_{R}(i d(R)) \rightarrow r A n n_{\left[\left[R^{S, \leq]]}\right.\right.}\left(i d\left(\left[\left[R^{S, \leq]]))}\right)\right.\right.\right.$ and $\psi:$ $l A n n_{R}(i d(R)) \rightarrow l A n n_{\left[\left[R^{S, \leq]}\right]\right.}\left(i d\left(\left[\left[R^{S, \leq}\right]\right]\right)\right)$ defined by $\phi(I)=I\left[\left[R^{S, \leq}\right]\right]$ and $\psi(J)=\left[\left[R^{S, \leq]] J}\right.\right.$ for every $I \in r A n n_{R}(i d(R))=\left\{r_{R}(U) \mid U\right.$ is an ideal of $\left.R\right\}$ and $J \in l A n n_{R}(i d(R))=\left\{l_{R}(U) \mid U\right.$ is an ideal of $R$, respectively. Obviously, $\phi$ is injective. In the following Theorem we show that $\phi$ and $\psi$ are bijective maps if and only if $R$ is $S$-quasi-Armendariz. This Theorem is a generalization of a result of Hashemi ([4, Proposition 2.1]) that generalizes a result of Hirano ([23, Proposition 3.4]).

Theorem 3.2. Let $R$ be a ring, $S$ a strictly ordered monoid and $\left[\left[R^{S, \leq}\right]\right]$ the generalized power series. Then the following are equivalent:

(1) $R$ is generalized power series quasi-Armendariz ring.

(2) The function $\phi: r A n n_{R}(i d(R)) \rightarrow r A n n_{\left[\left[R^{S, \leq}\right]\right]}\left(i d\left(\left[\left[R^{S, \leq}\right]\right]\right)\right)$ is bijective, where $\phi(I)=$ $I\left[\left[R^{S, \leq}\right]\right]$.

(3) The function $\psi: l A n n_{R}(i d(R)) \rightarrow l A n n_{\left[\left[R^{S, \leq]}\right]\right.}\left(i d\left(\left[\left[R^{S, \leq}\right]\right]\right)\right)$ is bijective, where $\psi(J)=$ $\left[\left[R^{S, \leq}\right]\right] J$.

Proof. $(1) \Rightarrow(2)$ Let $Y \subseteq\left[\left[R^{S, \leq}\right]\right]$ and $\gamma=\cup_{f \in Y} C(f)$. From Lemma 3.1 it is sufficient to show that $r_{\left[\left[R^{S, \leq]]}\right.\right.}(f)=r_{R} C(f)\left[\left[R^{S, \leq]}\right]\right.$ for all $f \in Y$. In fact, let $g \in r_{\left[\left[R^{S, \leq]]}\right.\right.}(f)$ and for any $h \in\left[\left[R^{S, \leq}\right]\right]$. Then $f h g=0$ and by assumption $f\left(u_{i}\right) \operatorname{tg}\left(v_{j}\right)=0$ for each $u_{i} \in$ $\operatorname{supp}(f), t \in R$ and each $v_{j} \in \operatorname{supp}(g)$. Then for a fixed $u_{i} \in \operatorname{supp}(f), t \in R$ and each $v_{j} \in$ $\operatorname{supp}(g), 0=f\left(u_{i}\right) \operatorname{tg}\left(v_{j}\right)=\left(c_{f\left(u_{i}\right)} c_{t} g\right)\left(v_{j}\right)$ and it follows that $g \in r_{R} \cup_{u_{i} \in \operatorname{supp}(f)} c_{f\left(u_{i}\right)} c_{t}\left[\left[R^{S, \leq}\right]\right]=$ $r_{R} C(f)\left[\left[R^{S, \leq]]}\right.\right.$. So $r_{\left[\left[R^{S, \leq}\right]\right]}(f) \subseteq r_{R} C(f)\left[\left[R^{S, \leq}\right]\right]$.

Conversely, let $g \in r_{R} C(f)\left[\left[R^{S, \leq}\right]\right]$, then $c_{f\left(u_{i}\right)} c_{t} g=0$ for each $u_{i} \in \operatorname{supp}(f), t \in R$. Hence, $0=\left(c_{f\left(u_{i}\right)} c_{t} g\right)\left(v_{j}\right)=f\left(u_{i}\right) \operatorname{tg}\left(v_{j}\right)$ for each $u_{i} \in \operatorname{supp}(f), t \in R$ and $v_{j} \in \operatorname{supp}(g)$. Thus,

$$
(f h g)(s)=\sum_{\left(u_{i}, v_{j}\right) \in X_{s}\left(f, c_{t} g\right)} f\left(u_{i}\right) \operatorname{tg}\left(v_{j}\right)=0
$$

and it follows that $g \in r_{\left[\left[R^{S, \leq]}\right]\right.}(f)$. Hence $r_{R} C(f)\left[\left[R^{S, \leq]}\right] \subseteq r_{\left[\left[R^{S, \leq]]}\right.\right.}(f)\right.$ and it follows that $r_{R} C(f)\left[\left[R^{S, \leq]}\right]=r_{\left[\left[R^{S, \leq}\right]\right]}(f)\right.$. So

$$
r_{\left[\left[R^{S, \leq]]}\right.\right.}(Y)=\cap_{f \in Y} r_{\left[\left[R^{S, \leq]]}\right.\right.}(f)=\cap_{f \in Y} r_{R} C(f)\left[\left[R^{S, \leq}\right]\right]=r_{R}(\gamma)\left[\left[R^{S, \leq}\right]\right] .
$$

$(2) \Rightarrow(1)$ Suppose that $f, g \in\left[\left[R^{S, \leq]}\right]\right.$ be such that $f\left[\left[R^{S, \leq}\right]\right] g=0$. Then $g \in r_{\left[\left[R^{S, \leq]}\right]\right.}(f)$ and by assumption $r_{\left[\left[R^{S, \leq]]}\right.\right.}(f)=\gamma\left[\left[R^{S, \leq}\right]\right]$ for some right ideal $\gamma$ of $R$. Consequently, $0=f c_{t} c_{g\left(v_{j}\right)}$ and for any $u_{i} \in \operatorname{supp}(f), 0=\left(f c_{t} c_{g\left(v_{j}\right)}\right)\left(u_{i}\right)=f\left(u_{i}\right) \operatorname{tg}\left(v_{j}\right)$ for each $u_{i} \in \operatorname{supp}(f), t \in R$ and 
$v_{j} \in \operatorname{supp}(g)$. Hence, $R$ is a generalized power series quasi-Armendariz ring. The proof of $(1) \Leftrightarrow(3)$ is similar to the proof of $(1) \Leftrightarrow(2)$.

Definition 3.3. A submodule $N$ of a left $R$-module $M$ is called a pure submodule if $L \otimes_{R} N \rightarrow$ $L \otimes_{R} M$ is a monomorphism for every right $R$-module $L$. By [1, Proposition 11.3.13], for an ideal I , the following conditions are equivalent:

(1) I is right s-unital;

(2) $R / I$ is flat as a left $R$-module;

(3) $I$ is pure as a left ideal of $R$.

Theorem 3.4. Let $R$ be a ring, $(S, \leq)$ a strictly totally ordered monoid. Then the following statements are equivalent:

(1) $r_{R}(a) R$ ) is pure as a right ideal in $R$ for any element $a \in R$;

(2) $r_{\left[\left[R^{S, \leq}\right]\right]}\left(f\left[\left[R^{S, \leq}\right]\right]\right)$ is pure as a right ideal in $\left[\left[R^{S, \leq}\right]\right]$ for any element $f \in\left[\left[R^{S, \leq}\right]\right]$.

In this case $R$ is an $S$-quasi-Armendariz ring.

Proof. Assume that the condition (1) holds. Firstly, by using the same method of the proof of Proposition 2.9 we can proved that $R$ is an $S$-quasi-Armendariz. Finally, by using Lemma 2.8 we can see that the condition (2) holds.

Conversely, suppose that the condition (2) holds. Let $a$ be an element of $R$. Then $r_{\left[\left[R^{S, \leq]]}\right.\right.}\left(a\left[\left[R^{S, \leq]}\right]\right)\right.$ is left $s$-unital. Hence, for any $b \in r_{R}(a R)$, there exists an element $f \in\left[\left[R^{S, \leq}\right]\right]$ such that $b f=b$. Let $f(0)$ be the constant term of $f$. Then $f(0) \in r_{R}(a R)$ and $f(0) b=b$. This implies that $r_{R}(a R)$ is left $s$-unital. Therefore condition (1) holds.

Let $R$ be a quasi-Baer ring and let $a \in R$. Then $l_{R}(R a)=R e$ for some idempotent $e \in R$, and so $R / l_{R}(R a) \cong R(1-e)$ is projective. Therefore a quasi-Baer ring satisfies the hypothesis of Theorem 3.4. Hence we have the following:

Corollary 3.5. Let $R$ be a ring, $(S, \leq)$ a strictly totally ordered monoid. Then a ring $R$ is a quasi-Baer ring if and only if $\left[\left[R^{S, \leq}\right]\right]$ is quasi-Baer ring.

\section{REFERENCES}

[1] B. Stenstrom, Rings of quotients, Springer-Verlag, 1975.

[2] C. Huh, Y. Lee and A. Smoktunowicz, Armendariz rings and semicommutative rings, Comm. Algebra, 30(2) (2002), 751-761.

[3] C. E. Rickart, Banach algebras with an adjoint operation, Ann. of Math., 47 (1946), 528-550.

[4] E. Hashemi, quasi-Armendariz rings relative to a monoid, J. Pure Appl. Algebra, 211 (2007), 374-382.

[5] G. A. Elliott, P. Ribenboim, Fields of generalized power series. Archiv d. Math, 54 (1990), 365-371.

[6] G. Mason, Reflexive ideals, Comm. Algebra, 9(17) (1981), 1709-1724. 
[7] G. F. Birkenmeier, J. Y. Kim and J. K. Park, A sheaf representation of quasi-Baer rings, J. Pure Appl. Algebra, 146 (2000), 209-223.

[8] G. F. Birkenmeier, J. Y. Kim and J. K. Park, On quasi-Baer rings, Contemp. Math, 259 (2000), 67-92.

[9] G. F., Birkenmeier, J. Y. Kim and J. K. Park, On polynomial extensions of principally quasi-Baer rings, Kyungpook Math. J, 40 (2000), 247-254.

[10] G. F. Birkenmeier, J.Y. Kim and J. K. Park, Principally quasi-Baer rings, Comm. Algebra, 29 (2001), 639-660.

[11] H. Tominaga, On s-unital rings, Math. J. Okayama Univ, 18 (1976), 117-134.

[12] K. Varadarajan, Noetherian generalized power series rings and modules. Comm. Algebra, 29(1) (2001a), 245-251.

[13] K. Varadarajan, Generalized power series modules. Comm. Algebra, 29(3) (2001b), 1281-1294.

[14] L. Zhao, X. Zhu and Q. Gu, Reflexive rings and their extensions, Math. Slovaca, 63(3) (2013), 417-430.

[15] P. P. Nielsen, Semicommutative and McCoy conditon, J. Pure Appl. Algebra, 2006, 298: 134-141.

[16] P. Pollingher, A. Zaks, On Baer and quasi-Baer rings, Duke Math. J, 37 (1970), 127-138.

[17] P. Ribenboim, Rings of generalized power series: Nilpotent elements, Abh. Math. Sem. Univ. Hamburg, 61 (1991), 15-33.

[18] P. Ribenboim, Noetherian rings of generalized power series. J. Pure Appl. Algebra, 79 (1992), 293-312.

[19] P. Ribenboim, Rings of generalized power series II: Units and zero-divisors. J. Algebra, 168 (1994), 71-89.

[20] P. Ribenboim, Semisimple rings and von Neumann regular rings of generalized power series, J. Algebra, 198 (1997), 327-338.

[21] R. M. Salem, Generalized Power Series over Zip and Weak Zip Rings, Southeast Asian Bull. Math, 37 (2013), 259-268.

[22] W. E. Clark, Twisted matrix units semigroup algebras, Duke Math. J, 34 (1967), 417-424.

[23] Y. Hirano, On annihilator ideals of a polynomial ring over a noncommutative ring, J. Pure Appl. Algebra, 168 (2002), 45-52.

[24] Z. K. Liu, Special properties of rings of generalized power series, Comm. Algebra, 32(8) (2004), 32153226.

[25] Z. Renyu, Uniserial modules of generalized power series, Bull. Iranian Math. Soc, 38(4) (2012), 947-954.

[26] Z. Renyu, A generalization of PP-rings and p.q.-Baer rings, Glasgow Math. J. 48 (2006) 217-229.

[27] Z. K. Liu, A note on principally quasi-Baer rings, Comm. Algebra, 30(8) (2002), 3885-3890. 